\title{
Early childhood pacifier use in relation to breastfeeding, SIDS, infection and dental malocclusion
}

\section{Grade of Recommendation}

These Grades of Recommendation have been based upon the JBI developed Grades of Effectiveness:

Grade A: Effectiveness established to a degree that merits application.

Grade B: Effectiveness established to a degree that suggests application.

Grade C: Effectiveness established to a degree that warrants consideration of applying the findings.

Grade D: Effectiveness established to a limited degree.

Grade E: Effectiveness not established.

\section{Information Source}

This Best Practice Information Sheet is based on a systematic review of research, published by Blackwell Publishing Asia and conducted by the Western Australian Centre for Evidence Based Nursing and Midwifery, a Collaborating Centre of the Joanna Briggs Institute. ${ }^{(1)}$ The primary references included in the systematic review on which this information sheet is based are available online at www.blackwellsynergy.com and to members of the Institute via the web site: www.joannabriggs.edu.au

\section{Background}

A pacifier is an object that is given to a baby to suck so that the baby feels comforted and stays quiet. Pacifiers, colloquially known as "dummies", "soothers", and "comforters" in the English speaking world, are used widely to soothe or calm a child who is crying. Pacifiers are also used to prevent the sucking of thumbs and other objects, and as an aid to weaning.

Reports of infants sucking objects appear as early as the late fifteenth century. Small linen bags filled with bread, milk and sugar were used to nourish and comfort children in the early nineteenth century. The first patent on the India rubber nipple which resembles the present day pacifier was recorded in 1845 while the practice of dipping the dummy into a variety of sweetening agents to make it a more effective pacifier was first described in1927.

Currently, pacifiers are made of latex or silicone and they come in several different shapes and sizes. The nipple may be long or short, with a ball shaped or flattened end. A shield is attached to the nipple to prevent swallowing or choking.

Pacifier use varies between cultures, societies and communities. The reasons why carers decide to use a pacifier are many and varied, based on culture, past practice, health care policy and advice, and occasionally research. As many child health nurses and midwives advise pacifier use as do not. This advice is not based on a consistent and coherent rationale, but rather often based on personal experience or the belief that it is simply a matter of parental choice. 
A preliminary review of the research indicates that the use of a pacifier may lead to negative effects, such as a shortened duration of breastfeeding, infection and dental malocclusion. On the other hand, pacifier use may reduce an infant's risk of Sudden Infant Death Syndrome (SIDS).

\section{Studies Included and Quality of Evidence}

The studies included in the review involved participants who were healthy term infants and healthy children up to the age of 16 years. Studies which focused on preterm infants, and infants and young children with serious illness or congenital malformations were excluded (however, some total population studies did include these children). As very few randomised controlled trials (RCTs) on pacifier use had been conducted, observational epidemiological designs, specifically prospective cohort studies and, in the case of SIDS research, case-control studies were included in this review. Purely descriptive and cross-sectional studies were excluded, as were qualitative studies and all other forms of evidence. These studies did not meet the requirement of appropriate temporal sequencing of events nor report estimates of the strength of association. The studies included in the final review had been published over 10 years from 1993 to 2003 . The great majority of literature was published in the 1990s, with substantial contributions made since in the breastfeeding literature. Twelve studies were cohort designs, six were case-control studies, and two were RCTs.

\section{The relationship between pacifier use and breastfeeding}

A comprehensive literature search shows that breastfeeding plays a fundamental role in child health and development. Breastfeeding leads to effective mother-infant bonding and human milk is the most appropriate nutrition for all infants. Pacifier use has been implicated as a barrier to breastfeeding for quite some time, leading to partial rather than exclusive feeding and/or a shorter duration. This review focuses on the accumulated evidence for a causal relationship between pacifier use and disrupted breastfeeding behaviour.

Ten studies met the inclusion criteria, comprising two RCTs and eight cohort studies. The research was conducted between 1995 and 2003 in a wide variety of settings (Australia, Brazil, Canada, Italy, New Zealand, Sweden, and the USA) involving research participants from diverse socio-economic and cultural backgrounds. Motherinfant pairs were recruited conveniently from hospitals and health clinics, and randomly from selected populations. Follow-up time ranged from four weeks until the cessation of breastfeeding which for one study was beyond the first year. Both the level of contact and the frequency of contact with the informant, the child's mother, differed widely.

Few studies were initiated expressly to investigate the relationship of pacifier use with breastfeeding. Therefore, pacifier use was defined and measured inconsistently. Both exclusivity and duration of breastfeeding were employed as outcome measures, but definitions also varied considerably. In addition, the difference between exclusive breastfeeding (breast milk being the only source of milk with infrequent supplements of water, juice or other fluids) and partial breastfeeding (a combination of breast milk and other infant or milk formulas with the addition of other supplements of water, juice etc) was not uniformly identified.

Completeness of follow-up was addressed, but missing data were not uniformly identified and explained. When comparisons were made between participants and nonparticipants there was some evidence of differential loss and a bias toward families in 
higher socio-economic groups. Multivariate analysis was undertaken in the majority of studies, with some including a large number of socio-demographic, obstetric, and infant covariates and others including just maternal age and education. Given the inconsistency of definition and measurement, the relationship between pacifier use and breastfeeding was expressed in many different ways and a meta-analysis was not appropriate.

\section{Summary of findings}

All but one study reported that pacifier use was associated with a reduction in breastfeeding duration or exclusivity. The data suggest that very infrequent use may not have any overall negative impact on breastfeeding outcomes. There are two possible explanations for the consistent finding of an association between pacifier use and negative breastfeeding outcomes. First, it is entirely plausible that pacifier use causes babies to breast feed less. The innate sucking reflex of the infant is satisfied by the pacifier, decreasing or eliminating the desire for contact with the nipple and breast. Second, pacifier use does not cause a reduction in breastfeeding; it is simply a marker for socio-economic, demographic, psychosocial, and cultural factors that determine both pacifier use and breastfeeding. An alternative argument that the cessation of breastfeeding leads to pacifier use has not, to date, been tested.

\section{The relationship between pacifier use and SIDS}

SIDS is a major classification of mortality in infants between one month and one year of age in Western industrialised countries. The aetiology of SIDS is poorly understood, however, epidemiological research has identified a number of factors and modifiable infant care practices which appear to increase or decrease the risk. Some of the practices reported to reduce the risk of SIDS include: breastfeeding, room-sharing and pacifier use.

Six published case-control studies met the criteria for inclusion. The research was conducted with information gathered between 1984 and 1999 in Norway, the UK, New Zealand, the Netherlands and the USA. Cases were infants who had died of SIDS. All controls were drawn from the community at a ratio to cases varying between one-to-one to four-to-one. In all but one study, controls were matched to cases on factors such as age, sex, time of birth and region. Information about pacifier use was obtained from a variety of sources including: hospital and antenatal records, death scene investigation, and interview and questionnaire.

In the majority of the studies the findings were determined by post-mortem. Pacifier use was again defined and measured somewhat inconsistently. Five studies referred to pacifier use in the "last" sleep for SIDS cases and an assigned "reference" sleep for the control, matched to the time of the case's death. All studies controlled for confounding factors by matching and/or using multivariate analysis. Generally, antenatal and postnatal factors as well as infant care practices and maternal, family, and socioeconomic issues were considered.

\section{Summary of findings}

With regard to pacifier use in the last sleep (SIDS case) or reference sleep (control), all five studies reporting multivariate results found significantly more controls (non-SIDS) used a pacifier compared with cases (SIDS). That is, pacifier use was associated with a reduced incidence of SIDS. The results indicate that the risk of SIDS for infants who did 
not use a pacifier in the last or reference sleep was at least twice, and possibly five times, that of infants who did use a pacifier.

Several causal mechanisms have been proposed to explain the finding of a relationship between pacifier use and the risk of SIDS, including the following: the presence of a pacifier may protect the infant's airway; pacifier sucking, or just the presence of a pacifier, may lessen the likelihood of apnoea; and pacifier use may reduce high risk infant sleep behaviours, such as a prone sleeping position. Most researchers and clinicians, however, are reluctant to actively promote the use of pacifiers in the absence of adequate knowledge regarding actual mechanisms related to pacifier use and SIDS.

\section{The relationship between pacifier use and infection}

It has been postulated that pacifier use was associated with gastro-intestinal, upper respiratory tract, lower respiratory tract infection and dental caries.

Eighteen research articles that purported to investigate the relationship between pacifier use and infection were reviewed. Of these, only four met the inclusion criteria. One cohort study examined the possible association between pacifier use and acute otitis media. Another cohort study looked at the association between pacifier use and dental infection leading to dental caries. Two studies utilised a birth cohort to investigate the association between pacifier use and a range of outcomes at different ages including respiratory symptoms, ear problems, gastro-intestinal symptoms and other symptoms of infection. In these two studies the outcomes were 'symptoms only', not medically diagnosed infections.

Of the four studies that met the selection criteria, three reported a positive association between pacifier use and infection. Conversely, the fourth study found no positive association between pacifier use at 15 months of age and a range of infections experienced between the ages of 6 and 18 months.

\section{$\underline{\text { Summary of findings }}$}

Given the limited number of studies available and the variability of results, no meaningful conclusions could be drawn.

\section{The relationship between pacifier use and dental malocclusion}

The relationship of pacifier use and dental malocclusion has been a subject of great interest to dentists and child health professionals for many years.

Forty-seven articles were identified that purported to investigate this relationship. Of these, five were cohort studies and one was a case-control study. There were no RCTs and the excluded articles were mostly case-series and cross-sectional designs. Not one of the cohort or case-control studies reported a measure of association, such as an estimate of relative risk. It was therefore not possible to include these studies in the final review.

\section{Summary of findings}

Due to the absence of adequate studies, no conclusions could be made. 


\section{Implications for Practice}

1. Breastfeeding: pacifier use in infancy is associated with a shorter duration and non-exclusivity. It is plausible that pacifier use causes babies to breast feed less, but a causal relationship has not been irrefutably proven.

2. SIDS: the evidence for a relationship between pacifier use and a reduction in risk for SIDS is consistent, while the exact mechanism of the effect is not well understood.

3. Infection: it was found that, due to the paucity of epidemiological studies, no meaningful conclusion can be drawn between pacifier use and infection.

4. Dental Malocclusion: it was found that due to the complete lack of adequate epidemiological studies, no findings could be made.

\section{Recommendation}

As breastfeeding confers an important advantage on all children and the incidence of SIDS is very low, it is recommended that health professionals generally advise parents against pacifier use, while taking into account individual circumstances (Grade B).

\section{Acknowledgement}

This Best Practice Information sheet was developed by the Western Australian Centre for Evidence Based Nursing and Midwifery (WACEBNM), a Collaborating Centre of the Joanna Briggs Institute with a review panel of community health nurses and researchers led by Ann Callaghan, Nurse Researcher, Telethon Institute for Child Health Research. The WACEBNM would like to acknowledge and thank the members of the review panel whose support, expert advice and commitment to the review proved to be invaluable throughout this activity.

Members of the review panel were:

Ann Callaghan RN, RM, BNurs(Hons)

Garth Kendall RN, BA(Psych), DipSocSci(Nursing), MPH, PhD

Christine Lock RN, RM, CHN, IBCLC, BSc(Nursing), MSc(Nursing)

Anne Mahony, RN, BNurs(Hons), RSCN(UKCC), PhD

Jan Payne SRN(UKCC), P Grad Dip(Hlth Admin), MSc(Pub Hlth)

Leanda Verrier RN, RM, CHN, MSc(Nursing)

Clinicians:

Dianne Bootle

Anna Bosco

Carolien Giele

Julie Holschier

Jane Hubble

Petrine Keech

Eileen McKeogh 
Kathleen McKeogh

Bev Nicholson

Ailsa Rothenbury

Megan Scully

Lorraine Tee

Marie Tyrell-Clarke

Kim Wedge

The professional backgrounds of the Review Panel and the Clinicians included community and child health nurses, registered nurses and didwives, and nurse researchers.

In addition this Best Practice Information Sheet has been peer reviewed by experts nominated by Joanna Briggs Institute collaborating centres.

\section{References}

1. Callaghan A, Kendall G, Lock C, Mahony A, Payne J, Verrier L. Association between pacifier use and breastfeeding, sudden infant death syndrome, infection and dental malocclusion. International Journal of Evidence-Based Healthcare 2005; 3(6):147-167. 